\title{
The feet of the altitudes of a simplex
}

\author{
Arthur Gut and Jörg Waldvogel \\ Arthur Gut received his Ph.D. from ETH Zürich with B. Eckmann. After postdoctoral \\ stays in the U.S.A. and Mexico he became a Gymnasium teacher at the Kantonsschule \\ Zürich-Oerlikon; since 2005 he is retired. His mathematical interests are geometry \\ and computer algebra systems in education and in applications. \\ Jörg Waldvogel received his Ph.D. in celestial mechanics from ETH Zürich. From \\ 1973 he held a position at ETH, first as the head of the Numerical Algorithms Group, \\ later as a professor, until his retirement in 2003. His research interests are numerical \\ mathematics, celestial mechanics, and number theory.
}

\section{Introduction}

The construction of the feet of the altitudes of a triangle or a tetrahedron is a standard task in elementary geometry or solid geometry. The planar case is of particular interest: The feet $F_{0}, F_{1}, F_{2} \in \mathbb{R}^{2}$ of the altitudes of a triangle with vertices $A_{0}, A_{1}, A_{2} \in \mathbb{R}^{2}$ form the so-called orthic triangle. This triangle is the solution of Fagnano's problem (1775), see, e.g., [1]: Among all triangles $P_{0}, P_{1}, P_{2}$ with $P_{k}$ on the side opposite to $A_{k}(k=0,1,2)$ or on its extension, find the triangle with minimal perimeter.

In $n \geq 2$ dimensions the computation of all feet $F_{0}, F_{1}, \ldots, F_{n} \in \mathbb{R}^{n}$ of a simplex in $\mathbb{R}^{n}$ from the coordinates of its vertices $A_{0}, A_{1}, \ldots, A_{n} \in \mathbb{R}^{n}$ is therefore of interest. In this note we describe a surprisingly compact algorithm for this task that is valid in every

Im Unterricht in Elementargeometrie gehören die Höhen im Dreieck zum Standardstoff. Die Verallgemeinerung auf das Tetraeder im Raum liefert gelegentlich Material für Maturitätsaufgaben, etwa Berechne den der Ecke A gegenüberliegenden Höhenfusspunkt $F_{A}$ im Tetraeder ABCD aus den Koordinaten der Eckpunkte. Es zeigt sich, dass es ein erstaunlich einfaches und kompaktes Verfahren für die simultane Berechnung aller Höhenfusspunkte eines $n$-dimensionalen Simplex gibt. Dieses funktioniert für alle Dimensionen $n \geq 1$ und beruht auf der Verwendung homogener Koordinaten und projektiver Räume. Nach einer kurzen Einführung dieser Begriffe werden zunächst alle Seitenflächen eines Simplex mit einer einzigen Matrixinversion berechnet. 
dimension $n \geq 1$. The basis of our approach is the use of homogeneous coordinates that will be reviewed in the next section. Then, the faces of the simplex will be represented by means of an inverse matrix. Finally, the feet are obtained by intersecting the altitudes with the faces. An elegant code in MATLAB [4] will be given at the end.

\section{Homogeneous coordinates}

In homogeneous coordinates the point $X=\left(X_{1}, X_{2}, \ldots, X_{n}\right)^{T} \in \mathbb{R}^{n}$ is represented by the $(n+1)$-tuple $\mathbf{x}=\left(x_{0}, x_{1}, \ldots, x_{n}\right)^{T} \in \mathbb{R}^{n+1} \backslash\left\{(0,0, \ldots, 0)^{T}\right\}$, where the superscript $T$ denotes transposition and

$$
X_{k}=\frac{x_{k}}{x_{0}}, \quad k=1, \ldots, n,
$$

for any $x_{0} \neq 0$. Any multiple $c \mathbf{x}$ of $\mathbf{x}$ with $c \neq 0$ represents the same point. A projective space is obtained by allowing $x_{0}=0:\left(0, x_{1}, \ldots, x_{n}\right)^{T}$ represents the infinitely remote point, or absolute point, in the direction of $\left(x_{1}, x_{2}, \ldots, x_{n}\right)^{T} \in \mathbb{R}^{n}$.

All points $X=\left(X_{1}, X_{2}, \ldots, X_{n}\right)^{T} \in \mathbb{R}^{n}$ satisfying the linear relation

$$
e_{0}+e_{1} X_{1}+\ldots+e_{n} X_{n}=0
$$

form an $(n-1)$-dimensional hyperplane $E \subset \mathbb{R}^{n}$. The elements $e_{k}$ of the $(n+1)$-tuple $\mathbf{e}=\left(e_{0}, e_{1}, \ldots, e_{n}\right)^{T} \in \mathbb{R}^{n+1} \backslash\left\{(0,0, \ldots, 0)^{T}\right\}$ are called the hyperplane coordinates, or Plücker coordinates, of $E$; again, any multiple $c \mathbf{e}$ of $\mathbf{e}$ with $c \neq 0$ represents the same hyperplane. Equation (2) with (1) yields the condition of incidence,

$$
\sum_{k=0}^{n} e_{k} x_{k}=0 \quad \text { or } \quad \mathbf{e}^{T} \mathbf{x}=0
$$

i.e. the hyperplane $E$ contains the point $X$ if and only if the dot product of the homogeneous vectors $\mathbf{e}$ and $\mathbf{x}$ vanishes.

From (2) it is seen that the vector $\left(e_{1}, e_{2}, \ldots, e_{n}\right)^{T} \in \mathbb{R}^{n}$ is orthogonal to every vector in the hyperplane $E$. Therefore, the direction of $\left(e_{1}, e_{2}, \ldots, e_{n}\right)^{T}$, i.e. its absolute point

$$
\mathbf{e}^{0}:=\left(0, e_{1}, e_{2}, \ldots, e_{n}\right)^{T} \in \mathbb{R}^{n+1}
$$

corresponds to the direction orthogonal to $E$. For a more detailed introduction to projective spaces and homogeneous coordinates see, e.g., [1] or [3].

\section{The faces of a simplex}

Let the $n+1$ vertices $A_{k}(k=0, \ldots, n)$ of a nondegenerate simplex in $\mathbb{R}^{n}$ be given by their homogeneous coordinates, e.g.

$$
\mathbf{a}_{k}=\left(1, A_{k 1}, A_{k 2}, \ldots, A_{k n}\right)^{T}, \quad k=0, \ldots, n,
$$


where $A_{k j}$ is the $j$-th Cartesian component of the position vector of the $k$-th vertex. Consider now the matrix

$$
A=\left(\mathbf{a}_{0}, \mathbf{a}_{1}, \ldots, \mathbf{a}_{n}\right) \in \mathbb{R}^{(n+1) \times(n+1)}
$$

whose columns are the homogeneous coordinates of the vertices of the simplex. Assuming that $A$ is invertible, we denote its inverse by $S^{T}$; hence

$$
S^{T} \cdot A=I \quad \text { or } \quad S:=\left(A^{-1}\right)^{T},
$$

where $I$ is the unit matrix of order $n+1$. By partitioning $S^{T}$ into its rows $\mathbf{s}_{k}^{T}=$ $\left(s_{k 0}, s_{k 1}, \ldots, s_{k n}\right)$ we have

$$
S^{T}=\left(\begin{array}{c}
\mathbf{s}_{0}^{T} \\
\vdots \\
\mathbf{s}_{n}^{T}
\end{array}\right) \quad \text { or } \quad S=\left(\mathbf{s}_{0}, \mathbf{s}_{1}, \ldots, \mathbf{s}_{n}\right),
$$

and (7) may be written as

$$
\mathbf{s}_{k}^{T} \mathbf{a}_{l}=\left\{\begin{array}{cc}
0, & k \neq l, \\
1, & k=l .
\end{array}\right.
$$

Therefore, as a consequence of (3), the hyperplane defined by the homogeneous vector $\mathbf{s}_{k}$ contains every vertex of the simplex except $A_{k}$; it is therefore the face opposite to $A_{k}$. Hence, we have the following

Theorem 1. Let $A \in \mathbb{R}^{(n+1) \times(n+1)}$ be the matrix containing in its $k$-th column the homogeneous coordinates of the vertex $A_{k}(k=0, \ldots, n)$ of a nondegenerate simplex in $\mathbb{R}^{n}$, $n \geq 1$. Then, the transpose of the inverse of $A, S:=\left(A^{-1}\right)^{T}$, contains the homogeneous coordinates of the face opposite to $A_{k}$ in its $k$-th column.

The matrix $A$ in the above theorem is guaranteed to be invertible: Assume that $A$ is singular; then we can express one of the columns of $A$, say the $k$-th column, as a linear combination of the remaining columns. Hence, the vertex $A_{k}$ lies in the subspace spanned by the rest of the vertices, and the simplex is degenerate, in contradiction with the hypotheses of the theorem.

\section{The feet of the altitudes}

In the following, we restrict ourselves to proper simplices, i.e. no vertices are at infinity. Therefore, the matrix $A$ defined in (6) contains no zeros in its first row. The $k$-th altitude of the simplex may be defined as the line connecting the vertex $A_{k}$ with the absolute point $\mathbf{s}_{k}^{0}:=\left(0, s_{k 1}, s_{k 2}, \ldots, s_{k n}\right)^{T}$ of the normal of the face opposite to $A_{k}$. In general, the line connecting two points $A, B$ (given by their homogeneous coordinates $\mathbf{a}, \mathbf{b}$, respectively) is the set of points $\mathbf{c}=\lambda \mathbf{a}+\mu \mathbf{b}$ with $\lambda, \mu \in \mathbb{R},(\lambda, \mu) \neq(0,0)$. For the $k$-th altitude we may choose $\mathbf{c}=\mathbf{f}_{k}:=\mathbf{a}_{k}-\mu_{k} \mathbf{s}_{k}^{0}, \mu_{k} \in \mathbb{R}$, for simplicity. Its foot satisfies $\mathbf{s}_{k}^{T} \mathbf{f}_{k}=0$ which implies

$$
\mu_{k}=\frac{\mathbf{s}_{k}^{T} \mathbf{a}_{k}}{\mathbf{s}_{k}^{T} \mathbf{s}_{k}^{0}} .
$$


Evaluating the numerator by means of (9) and using the explicit expression for the denominator yields

$$
\mathbf{f}_{k}=\mathbf{a}_{k}-\mu_{k} \mathbf{s}_{k}^{0} \quad \text { with } \quad \mu_{k}=\frac{1}{\sum_{j=1}^{n} s_{k j}^{2}}, k=0,1, \ldots, n
$$

for the foot of the $k$-th altitude. In order to obtain all feet at once we consider the $n+1$ first equations of (11) as the columns of a single matrix equation,

$$
F=A-S^{0} \cdot \operatorname{diag}\left(\mu_{0}, \mu_{1}, \ldots, \mu_{n}\right)
$$

where $F$ is the matrix with the homogeneous coordinates of the feet as its columns. Furthermore, $\operatorname{diag}\left(\mu_{0}, \ldots, \mu_{n}\right)$ is the diagonal matrix with $\mu_{0}, \ldots, \mu_{n}$ as its diagonal elements. Finally, $S^{0}=\left(\mathbf{s}_{0}^{0}, \mathbf{s}_{1}^{0}, \ldots, \mathbf{s}_{n}^{0}\right)$, the matrix of the absolute points of the vectors orthogonal to the faces, is obtained from $S$ by zeroing out the first row and may be written as

$$
S^{0}=\operatorname{diag}(0,1, \ldots, 1) \cdot\left(A^{-1}\right)^{T}
$$

as a consequence of (7). We therefore have established

Theorem 2. Let $A \in \mathbb{R}^{(n+1) \times(n+1)}$ be the matrix containing in its $k$-th column the homogeneous coordinates of the vertex $A_{k}(k=0, \ldots, n)$ of a nondegenerate proper simplex in $\mathbb{R}^{n}, n \geq 1$. Then $S^{0}$ defined in (13) is the matrix of the absolute points of the vectors orthogonal to the faces, and $F$ defined in (12), with $\mu_{k}$ from (11), has the homogeneous coordinates of the feet of the altitudes of the simplex as its columns.

In the case of dimension $n=1$, when the simplex is a line segment, the algorithm simply exchanges the boundary points.

Remark. The use of projective spaces and homogeneous coordinates is widespread when it comes to deal with simplices. E.g., in [2] it can be seen how proofs of theorems get shortened and how the duality principle of projective geometry can enter the calculations.

\section{An elegant implementation in MATLAB}

The concise algorithm (13), (12) may be coded in the language of MATLAB [4] as follows below. MATLAB is particularly well suited owing to its powerful instructions for the operations of matrix algebra.

\% Given data: columns of $A$ are the homogeneous coordinates

of the vertices of the simplex. Example in 2 dimensions:

$$
A=\left[\begin{array}{rll}
1 & 1 & 1 \\
-3 & 3 & 1 \\
0 & 1 & 4
\end{array}\right] ;
$$


\% Algorithm

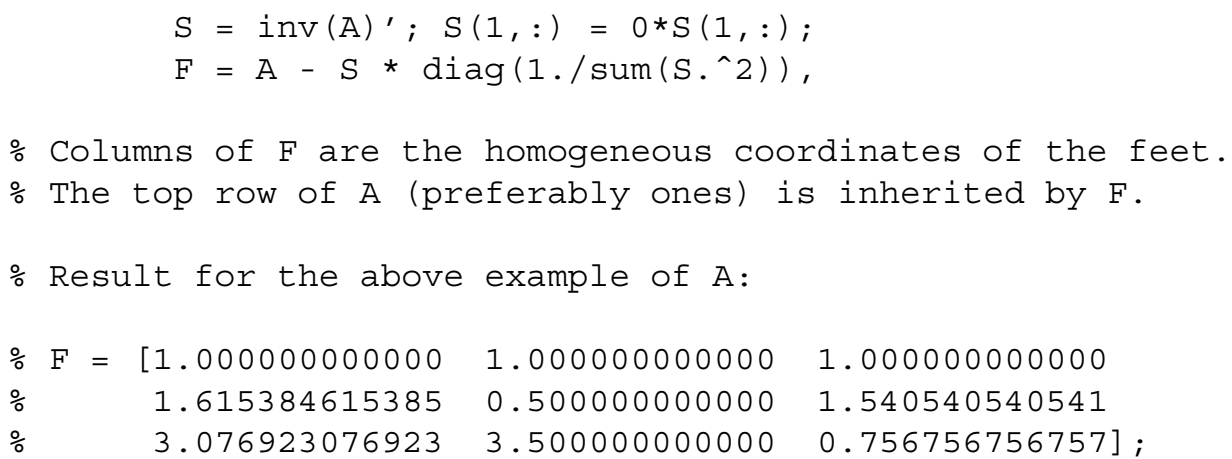

Comments on the code of the algorithm. We use the symbol $\mathrm{S}$ for the matrix $S^{0}$. The prime causes transposition of a real matrix. The second statement replaces the top row of the current $\mathrm{S}$ by a row of zeros. The operator diag fills its argument vector into a diagonal matrix. The sequence 1 . / sum (S.^2) computes all values $\mu_{k}$ for $k=0,1, \ldots, n$ of equation (11) by summation over the columns of the matrix of the squared elements of $S^{0}$.

\section{References}

[1] Coxeter, H.S.M.; Greitzer, S.L.: Zeitlose Geometrie. Klett, Stuttgart 1990. Original edition: Geometry Revisited. Random House, New York 1967.

[2] Gerber, L.: Associated and perspective simplices. Trans. Amer. Math. Soc. 201 (1975), 43-55.

[3] Kowalsky, H.-J.; Michler, G.O.: Lineare Algebra. Walter de Gruyter, Berlin, New York 2003.

[4] The MathWorks. URL http: / /www. mathworks. com

Arthur Gut

Kantonsschule Zürich-Oerlikon

Switzerland

e-mail: a.gut@ksoe.ch

Jörg Waldvogel

Seminar for Applied Mathematics

ETH Zürich

Switzerland

e-mail: waldvogel@math.ethz.ch 